\title{
A computer simulation study on the causal relationship between walking and physical malfunctions in older adults
}

\author{
Kazunori $\operatorname{HASE}^{1} *$ \\ ${ }^{1}$ Department of Mechanical Science and Engineering, Graduate School of Engineering, Nagoya University,
} Furocho, Chikusaku, Nagoya, 464-8603 Japan

Received 6 April 2006; accepted 6 September 2007

\begin{abstract}
The purpose of the present study was to investigate the causal relationship between walking pattern and physical malfunction using a computer simulation method with a biomechanical walking model. In this computer simulation, the musculoskeletal system was represented by a three-dimensional 14-rigid-link model and 60 muscle models. Muscular forces were controlled by a neuronal system model consisting of 16 pairs of neural oscillators. In the computational experiment, five types of walking models were constructed: an intact model, a delayed response model, a weak muscle model, an inclined posture model, and a joint contracture model. These malfunction factors were hypothesized to have a causal relationship with walking characteristics in older adults. The simulation revealed that the delayed response in the neuronal system was primarily related to walking stability. In addition, the weakening of muscles was strongly related to the reduction of the walking step length. The inclined posture and joint contracture also influenced the walking pattern, but not significantly. The use of such a computer simulation method is essential in order to clarify the causal relationship between body function and walking pattern in older adults.
\end{abstract}

Key words: neuromusculoskeletal system, walking factors, stability, computational experiment

\section{Introduction}

In older adults, body function and walking pattern change with age. In general, with increased age, walking speed becomes slower and step length becomes shorter (Murray et al., 1969). The biomechanical factors that characterize walking motion have been statistically analyzed in a number of studies, in which motion data were obtained experimentally from older adults (Kaneko et al., 1990; Nishizawa et al., 2000). Epidemiological studies also provide useful suggestions for the investigation of the causes of risk of falling and unstable walking. Furuna et al. (1998) elucidated the significant relation between maximum walking speed and risk of falling in older adults. Clarification of the biomechanical relationship between aging and human locomotion is important for anthropological science. For walking rehabilitation, it is also essential to identify and restore any physical malfunctions related to the risk of falling of unstable walking. However, it is difficult to directly measure these body functions with a non-invasive method, particularly in older adults. Moreover, several body functions are reduced simultaneously with age, so it is not always easy to identify the

From the International Symposium "Growth, Aging, and Motor Performance", 59th Annual Meeting of the Anthropological Society of Nippon, Nov. 2005

* Corresponding author. e-mail: kazunori.hase@mech.nagoya-u.ac.jp phone: +81-52-789-5582; fax: +81-52-789-5582

Published online 14 November 2007

in J-STAGE (www.jstage.jst.go.jp) DOI: 10.1537/ase.060406 causal relationship between a particular physical malfunction and walking performance.

In order to overcome such limitations, a computer simulation method for human walking has been developed (Hase and Yamazaki, 2002; Hase et al., 2003). This method allows conditions that are difficult to realize experimentally using actual human subjects to be examined by computer simulation. Therefore, this method has various possible applications to biomechanical studies, such as computational clarification of walking dysfunction factors and quantitative prediction of functions to stabilize human movement.

The purpose of the present study is to investigate the biomechanical relationship between walking and the malfunction of the physical body in older adults by using a three-dimensional neuromusculoskeletal model of human walking. In computer simulations, a specific body function was examined as a potential cause, and the effect on walking was then determined by motion synthesis using the mathematical model. Biomechanical factors related to aging, such as weakened muscle force, were investigated using the above methodology.

\section{Methods}

Neuromusculoskeletal model

This section outlines the proposed computer simulation model of human walking. The model has been described in detail in previous studies (Hase and Yamazaki, 2002; Hase et al., 2003).

The inertial properties of the entire human body were represented by a three-dimensional, 14-rigid-link system, as 


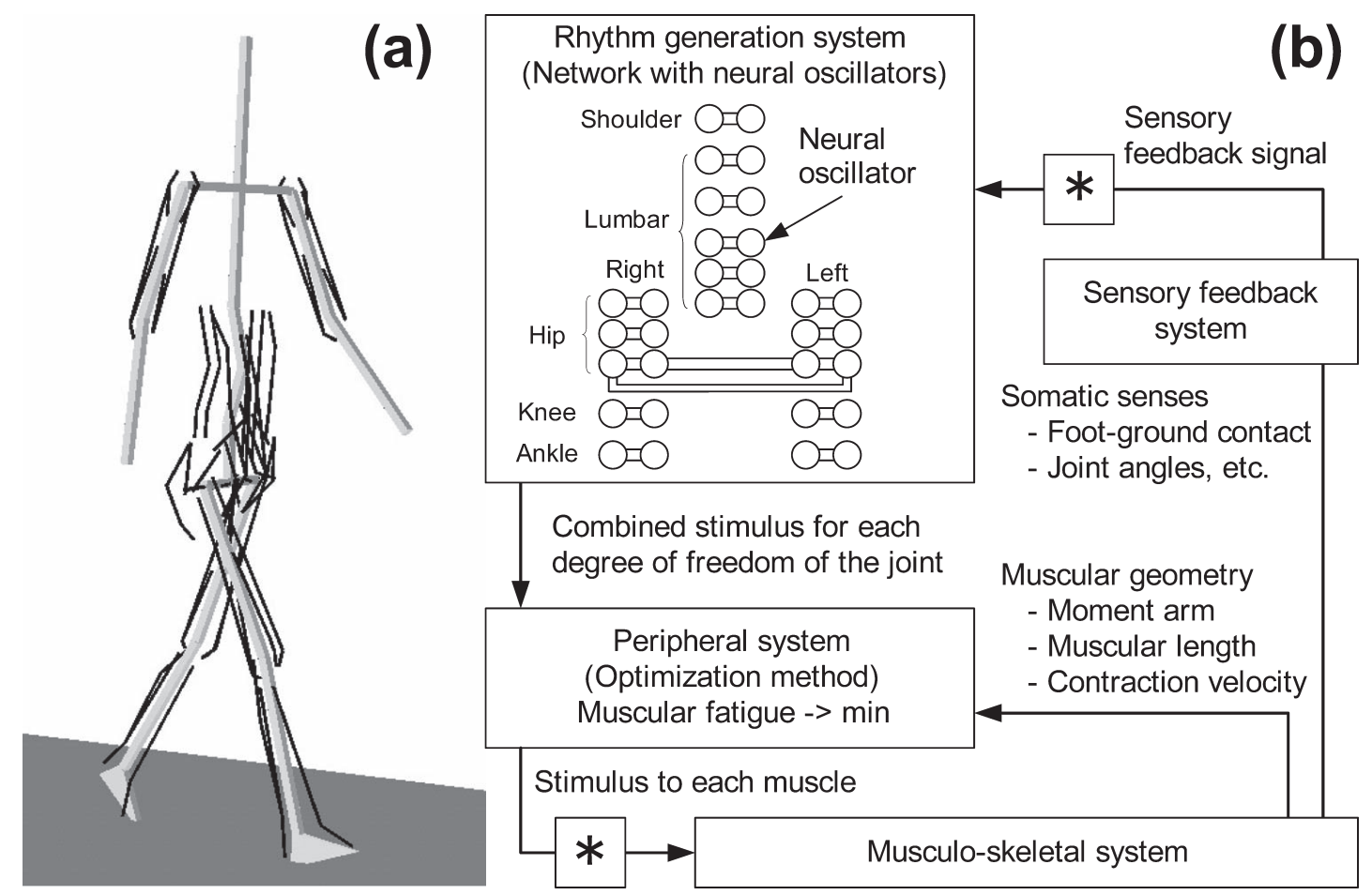

Figure 1. Neuromusculoskeletal model for human walking. (a) Three-dimensional model of the musculoskeletal system. The shaded thin boxes abstractly represent the rigid link system of the entire body. The bold lines indicate the muscular models represented by a series of line segments. (b) An outline of the neuronal model. The * symbols denote the delay elements that were considered only in the delayed response model. See text for details.

shown in Figure 1a. These links include the feet, the calves, the thighs, the pelvis, the lower part of the lumbar, the upper part of the lumbar, the thorax including the head and the neck, the arms, and the forearms. The hip joints and the lower lumbar joint (the joint between the lower lumbar and the pelvis segments, roughly corresponding to the lumbosacral joint) have three degrees of freedom of rotation. All other joints have one degree of freedom. A visco-elastic passive moment acts on each joint to represent the influence of soft tissue. The interaction between foot and ground was modeled as a combination of springs and dampers. The body dynamic model is driven by 60 muscle models for the entire body, as shown in Figure 1a. The geometric arrangement of each muscle was represented as a series of line segments, the direction of which changes according to the joint angle. The mechanical properties of each muscle are represented by a combination of the force-velocity relationship and the force-length relationship. Energy consumption, including heat production in the muscle, was calculated based on tension generation, and the length and contraction velocity of the muscle.

Central pattern generators (CPGs) are well-known systems that generate basic neural rhythms for locomotion. The rhythm generation system was modeled referring to Taga et al. (1991) and Taga (1995) as a recurrent network system consisting of neural oscillators, as shown in Figure 1b. A pair of neural models is used to model each degree of freedom of the joint. The pair of models generates the neuronal stimulus combined for each degree of freedom by receiving a nonspecific stimulus from the higher center (the neuronal system that is roughly equivalent to the brain stem, which activates the spinal cord and outputs a nonspecific constant parameter) and feedback signals from somatic senses, such as joint angles and foot-ground contact. Each neural oscillator is mathematically expressed by two differential equations. The neuronal model consists of 16 pairs of neural oscillators. In addition, the peripheral system model divides the combined neuronal stimulus from the neural oscillator into the neuronal stimulus to each muscle. The model corresponds to a macroscopic model of reciprocal innervations. The model is mathematically represented as a static optimization problem.

The neuronal system model contains many parameters that must be determined. The neuronal and mechanical properties of the model were modified by a numerical search method so as to achieve the desired walking pattern. The calculation flow was as follows: (1) the walking pattern was generated using the simulation model; (2) the evaluative criteria were calculated based on the generated walking motion; (3) the neuronal parameters were infinitesimally modified by an optimization method to improve the results for the evaluative criteria; and (4) these processes were repeated. In order to generate a normal walking pattern, a hybrid criterion between the energy efficiency for locomotion and motion smoothness was employed as the evaluative criterion, $C$ :

$$
C=\frac{1}{S+\omega D} \rightarrow \max
$$


where $S$ is the specific power for locomotion, $D$ is the rate of change of the muscle forces, and $\omega$ is the weighting coefficient. The specific power, $S$, for locomotion was defined by the following equation, which evaluates the energy efficiency for level locomotion (Ralston, 1976):

$$
S=\frac{\text { Metabolic Energy }}{\text { Body Mass } \cdot g \cdot \text { Distance Traveled }}
$$

where the metabolic energy was calculated from the muscle mechanical models in the walking simulator, and $g$ is gravitational acceleration. The rate of change of the muscle forces, $D$, was also calculated using the muscle forces simulated by the model, which evaluates the smoothness of the motion. Here, lower values of $S$ and $D$ indicate better or more efficient locomotion, so that the reciprocal was employed in the criterion that should be maximized.

The walking pattern and ground reaction forces simulated by the proposed model accurately represented the characteristics of normal walking in actual humans. The simulated muscle forces also agreed qualitatively with experimental electromyography (EMG) patterns measured in actual humans. The Appendix presents some detailed simulated data that were published in a previous paper (Hase and Yamazaki, 2002). This simulation model has been applied to various research fields, such as rehabilitation engineering (Hase and Obuchi, 2002) and sports science (Hase and Yokoi, 2002).

\section{Hypothesized walking models}

Based on the proposed model, various walking models were constructed in which the biomechanical factors related to aging were varied. That is, the following five types of walking models were used in the computational experiment: an intact model, a delayed response model, a weak-muscle model, an inclined posture model, and a joint contracture model. Each walking model included one independent factor that was believed to be a major factor in unstable walking, increasing the risk of falling in older adults. Detailed explanations of these models are given below.

\section{Intact model}

The intact model is a standard model, as mentioned above.

\section{Delayed response model}

The neuronal system in humans has a number of delay properties, such as muscular activation, and efferent and afferent paths. The delay time becomes more than $100 \mathrm{~ms}$ in some types of neurons. Moreover, the delayed response during walking was previously reported to be significantly different between younger and older adults (Hase et al., 2002). In the mechanical control theory with a feedback loop, adding a delay element makes the control of the system more difficult. Therefore, a relation between walking stability and such delay properties is hypothesized. The neuronal system in this simulation model has both the efferent path from the neural oscillators to the muscular model and the afferent path from the somatic receptors to the neural oscillators, as shown in Figure 1b. To construct the delayed response model, delay elements with the same delay time were inserted into both neuronal paths, as indicated by the asterisk symbols in
Figure $1 \mathrm{~b}$. The delay time was set to $4 \mathrm{~ms}$. This value was the longest delay at which the model was able to walk, although, compared to that in the actual nervous system, this time is very short. This problem will be discussed later herein.

\section{Weak muscle model}

Reduced muscle force and function is a general phenomenon that is characteristic of aging. In order to simulate the reduced muscle force in the mathematical model, the upper limit of the muscle force was set, and threshold processing was added to the muscle model; that it, the generating muscle force was restricted not to be over than the limit. The upper limit was set to $45 \%$ for the maximum muscle force of the intact model. The upper limit value of $45 \%$ was decided as the minimum value; that is, if the upper limit was set to below $45 \%$, the model could not walk.

\section{Inclined posture model}

As aging progresses, the upright posture becomes bent, causing an inclined posture effect. The reason for this inclined posture is the contracture of the spine, including muscles and ligaments. Such changes in the upright posture of older adults was thought to cause unstable walking because the location of the center of gravity of the upper body moves forward and the rotational movement of the pelvis is restricted if the upper body is bent forward. Therefore, the present study examines the influence of inclined posture on walking by simulation. The trunk segments were controlled to maintain a neutral posture that was predetermined for all of the simulation models. In the inclined posture model, the neutral joint angles in the thorax segment were changed so as to affect an inclined posture approximately $14^{\circ}$ from the vertical axis. This incline angle was decided as the maximum angle; that is, if the incline angle was set to be larger than $14^{\circ}$, the model could not walk.

\section{Joint contracture model}

Another cause of the malfunction of body dynamics properties in older adults is thought to be joint contracture (arthrogryposis). In general, joint movement loses it smoothness, and the range of the joint motion becomes narrower with age because of the contracture of the muscles and ligaments around the joint. Each joint in the simulation models has a nonlinear viscoelastic property representing passive joint resistance. In the joint contracture model, the magnitudes of the elastic properties of the joints were set to be five times larger than those of the intact model. The ratio of magnitudes was decided as the maximum; that is, if the magnitudes were set to be larger than five times, the model could not walk.

\section{The rate of parameter change in each hypothesized walking} model

As mentioned above, the rate of parameter change in each hypothesized model was determined from a computational point of view as the upper/lower limit of the parameter change at which the model could walk or not. Therefore, these rates did not correspond to the rate of change in actual humans with aging. One reason why this study employed such a computational limit on the rate of change of parame- 
ters was to clarify the influence of changes in parameters. A small rate of change will not influence the walking pattern of the model because the model, like a human, has the ability to stabilize its walking pattern against a perturbation. The other reason was a lack of the experimental data corresponding to the simulation model. In addition, the characteristics of aging vary widely, and it is difficult to extract the significant characteristics from experimental data with wide individual variations.

\section{Evaluation of walking pattern}

The neuronal parameters of each walking model were adjusted to walk continuously for at least six steps, and to maximize the evaluation criterion for locomotion. Although the basic structure of the neuronal control system was identical for all hypothesized models, the walking pattern obtained was adapted to the biomechanical condition of each model, because the neuronal parameters were adjusted by the numerical search method as mentioned before.

The locomotion pattern was then evaluated based on the following basic walking factors: walking speed, walking step length, walking cycle, specific power for locomotion, and walking stability. The specific power, $S$, in equation (2) was chosen as the basic walking factor, rather than the evaluative criterion, $C$, in equation (1), because $C$ is a hybrid criterion and does not have a precise physical dimension, whereas $S$ is more important than the rate of change of the muscle forces, $D$. It is difficult to quantify the stability performance of the walking pattern using only the obtained walking pattern. Therefore, in the present study, a slipping perturbation was added to the walking model in order to evaluate the stabilization ability. The slipping perturbation was realized by forcing the right foot to move forward at a speed of $1.0 \mathrm{~m} / \mathrm{s}$ for $0.2 \mathrm{~s}$ after the foot touched the ground. The walking stability was judged based on whether the model could walk continuously in the presence of the slipping perturbation.

\section{Results}

Figure 2 shows the walking patterns obtained from the hypothesized walking models, as well as the values of the basic walking factors calculated from each model. Figure 3 shows the basic time-series data from each model: the

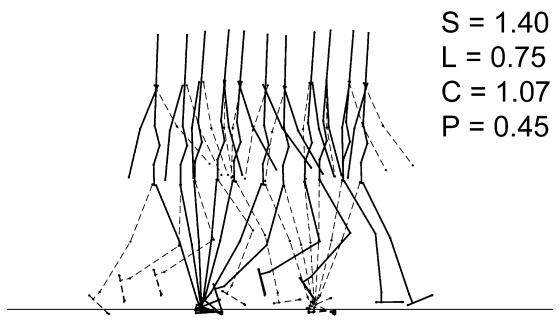

(a) Intact

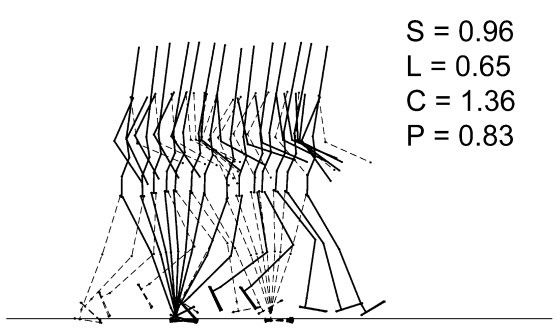

(b) Delayed response

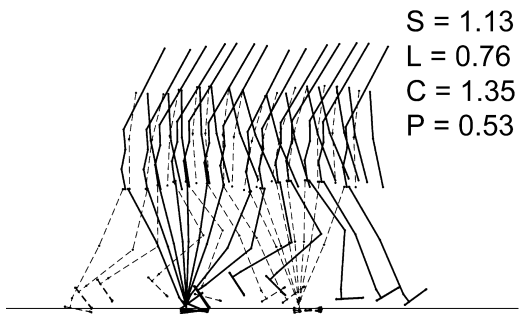

(d) Inclined posture

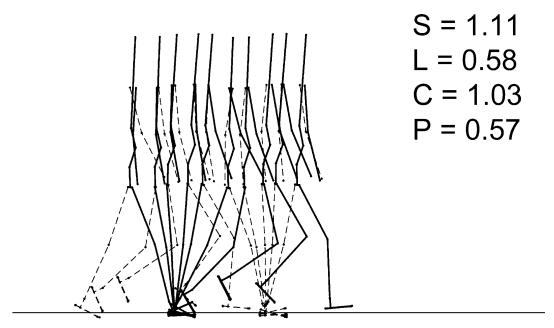

(c) Weak muscle

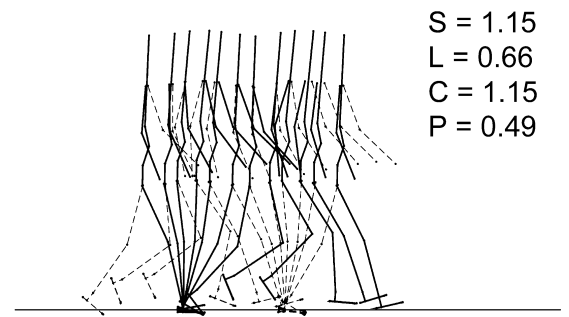

(e) Joint contracture

Figure 2. Simulation results of the hypothesized walking models: (a) intact model, (b) delayed response model, (c) weak muscle model, (d) inclined posture model, (e) joint contracture model. These stick pictures show the walking patterns of one cycle from right-foot contact to the next contact. Each stick picture is traced at $0.1 \mathrm{~s}$ intervals. The movement of the right upper and lower extremities was illustrated by dashed lines in this figure. Also shown in each figure are the values of the basic walking factors: $\mathrm{S}$ is the walking speed $(\mathrm{m} / \mathrm{s}) ; \mathrm{L}$ is the step length $(\mathrm{m})$; C is the walking cycle (s); and $\mathrm{P}$ is the specific power for locomotion (dimensionless). 
(1)

Intact
(2)

Delayed response
(3)

Weak muscle
(4) Inclined posture
(5)

Joint contracture

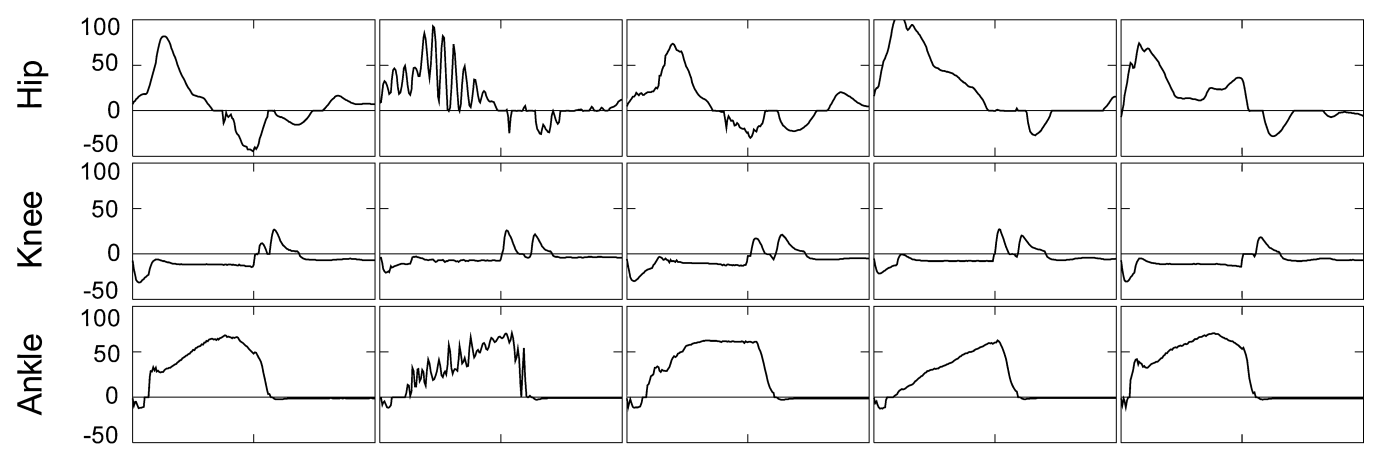

(a) Joint moments $[\mathrm{Nm}]$

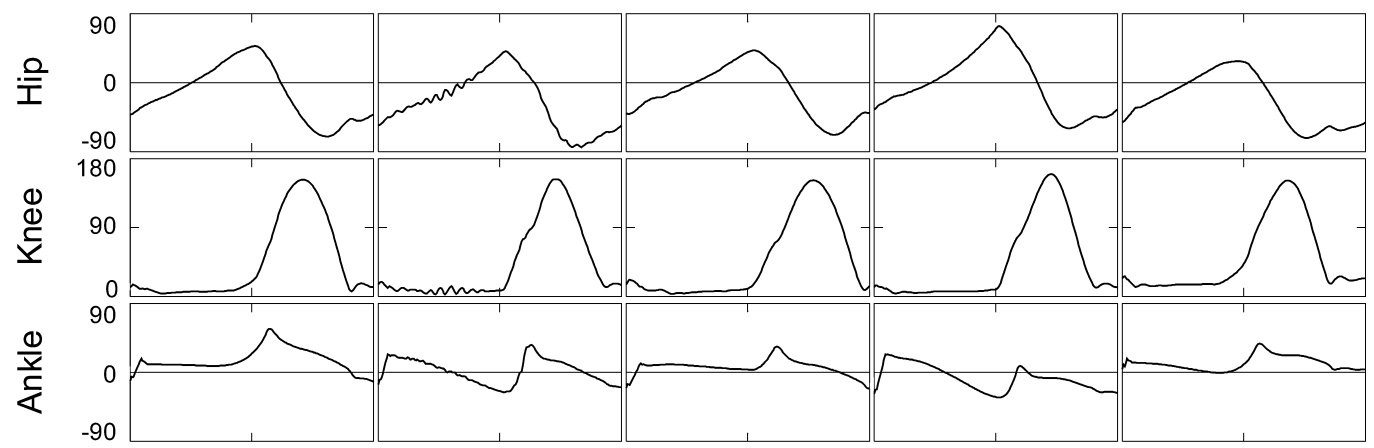

(b) Joint angles [degree]

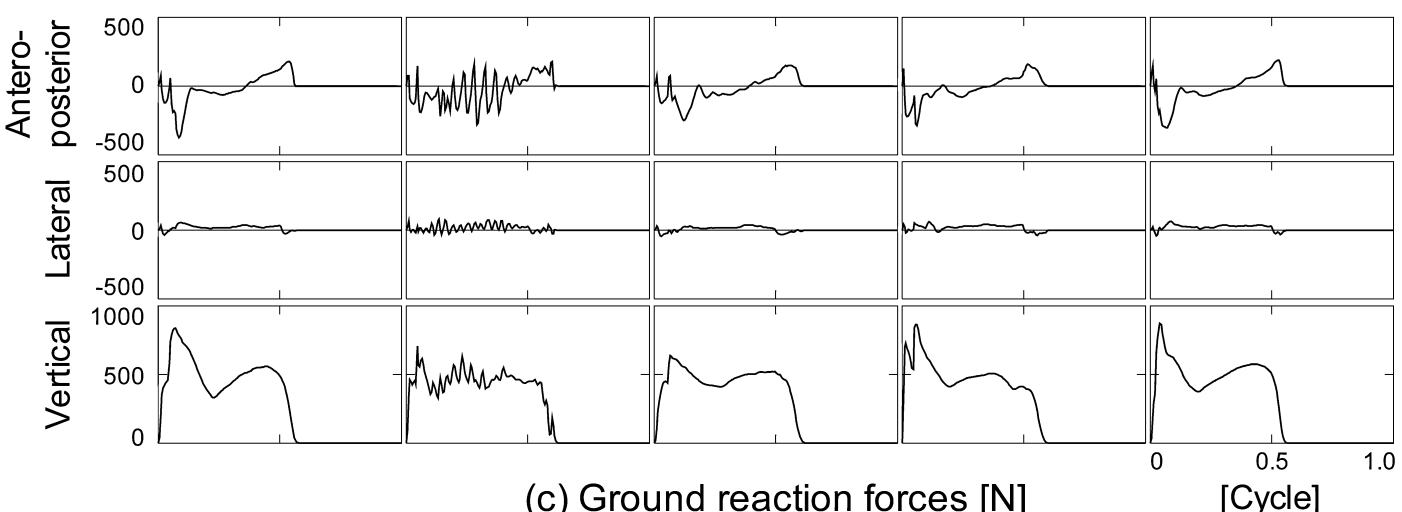

Figure 3. Time-series data obtained from the hypothesized models. Serial graphs on each column show the data from each hypothesized model: (1) intact model, (2) delayed response model, (3) weak muscle model, (4) inclined posture model, (5) joint contracture model. The same physical data located on each line of this figure: the three lines at the top of this figure are the joint moments $(\mathrm{Nm})$ at the hip, knee, and ankle joints; the three lines at the middle are the joint angles (degrees) at the hip, knee, and ankle joints; the three lines at the bottom are the anteroposterior, lateral, and vertical ground reaction forces $(\mathrm{N})$. The positive values of the joint moment and angle show clockwise movements. For all of the graphs, horizontal axes indicate time normalized by one walking cycle period, where 0 and 1 indicate the points at which the foot touches the ground.

ground reaction forces, joint angles and joint moments on the main joints. As shown in these figures, the walking pattern simulated by the intact model accurately represented the characteristics of actual human walking, and the other walking patterns by the hypothesized models were changed ac- cording to the model conditions. For example, the stride length in the weak muscle model was shorter and its walking cycle was longer. As a result, its walking speed was slower than that of the intact model. For the time-series data shown in Figure 3, the simulation results of the delayed response 
model included high-frequency vibrations. For the weak muscle model, the peaks of the ground reaction forces and joints moments decreased. For the inclined posture model, the extensor moment at the hip increased.

Figure 4 shows the walking patterns when the slipping perturbation was added for the purpose of evaluating the stability of walking. When the perturbation was added to the intact model, the model could walk continuously in the pres-

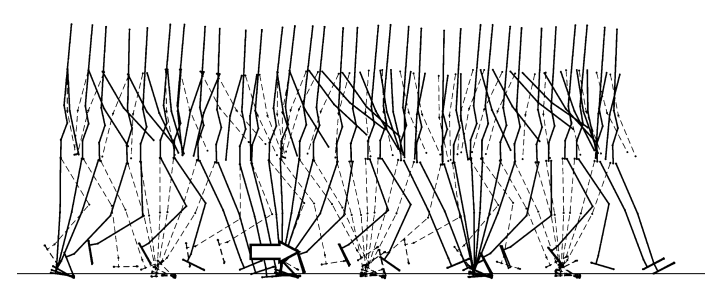

(a) Intact

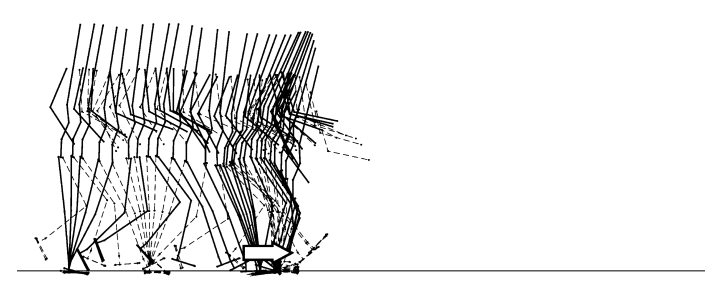

(b) Delayed response

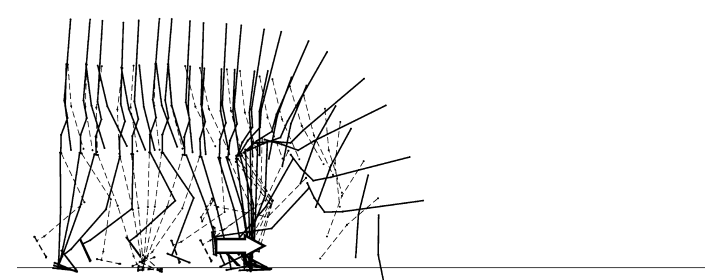

(c) Weak muscle

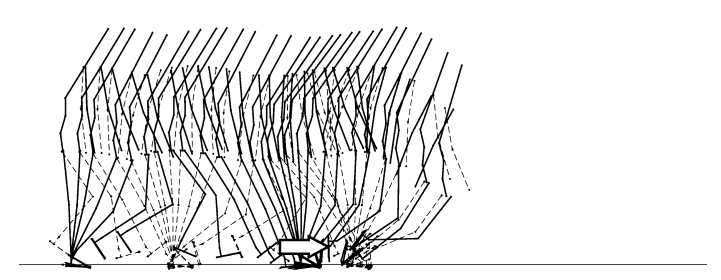

(d) Inclined posture

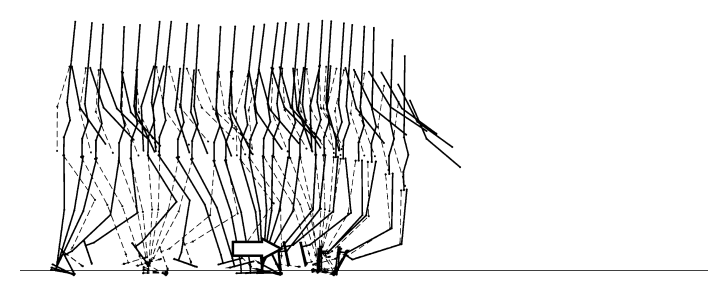

(e) Joint contracture

Figure 4. Assessment of walking stability using the computer simulation models and slip perturbation. At the beginning, in the second walking cycle in the simulation, a slip stimulus was introduced to the right foot, which is represented by solid lines. Arrows indicate the slip perturbation. Each stick picture was drawn as described in Figure 2. ence of the perturbation, as shown in Figure 4a. However, when the same perturbation was added to the other models, these models could not walk and fell down, as shown in Figure $4 b-e$.

Figure 5 shows the results of walking factors for the hypothesized models. These factors were normalized by those of the intact model. This figure clearly shows a relationship between the malfunction of physical body properties and the walking pattern.

\section{Discussion}

\section{Causal relationship between walking and physical mal- functions}

Compared to the intact walking model, the hypothesized models were characterized as follows. Malfunctions of the physical body reduced the walking speed in all of the hypothesized models compared to the intact model. Malfunctions also reduced the walking step length in the delayed response, weak muscle, and joint contracture models, but the walking step length was increased in the inclined posture model. The malfunctions increased the walking cycle in the delayed response and the inclined posture models, but no significant difference was observed between the intact model the weak muscle and joint contracture models. The malfunctions increased the specific power for locomotion in all of the hypothesized models. In particular, a significant increase in specific power was observed in the delayed response model. Finally, the malfunctions reduced the walking stability in all of the hypothesized models, as shown in Figure 4. Using such a simulation model clarified the causal relationship between the dynamic properties of the body and the generated walking pattern. The malfunctions hypothesized in the present study were all related to factors that made the walking pattern unstable and inefficient.

The relationship between malfunctions and unstable walking, as shown in Figure 4, is self-evident in a sense, because the physical parameters in the hypothesized models were set close to the limits, such that the model could not walk continuously. Therefore, the hypothesized models easily toppled due to a mechanical perturbation. These computational experiments simply mean that such a tendency was confirmed, and that such malfunctions in the hypothesized models did not contribute to any improvement in walking stability. In addition, all of the hypothesized models fell forward even though the forward slipping perturbation was added. One reason is that the perturbation was so small that the model could walk for one or two steps. Another reason is that the model was forced to walk continuously even after the perturbation was added, even though an actual human may stop walking or take a protective stance.

It is important to determine the most significant physical parameter for unstable, inefficient walking in older adults. However, these simulation results did not indicate this directly, because the dimensions of the hypothesized malfunctions are different and the rate of change hypothesized in the simulation did not always represent the actual rate of change with age as mentioned before. Therefore, in this section, the problem is discussed qualitatively.

First, the delay time of $4 \mathrm{~ms}$ that was used in the delayed 


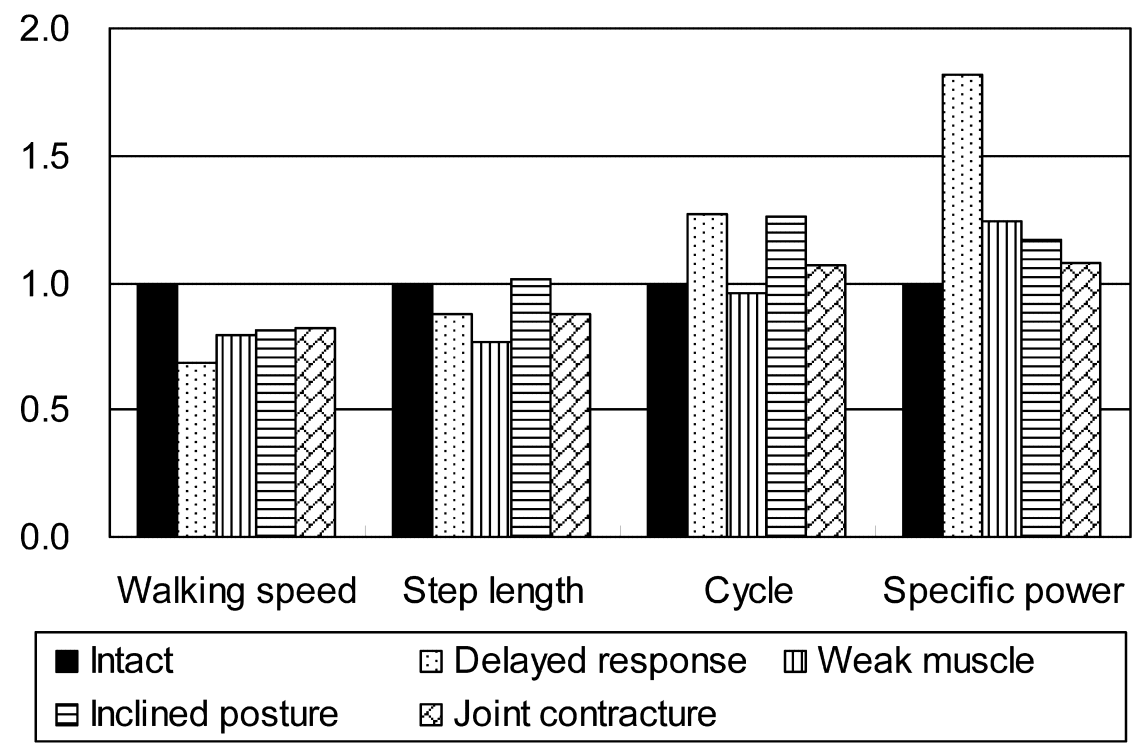

Figure 5. Comparison of walking factors. These parameters were normalized by those of the intact model.

response model was very short compared to that in the actual nervous system. The delay in the human leg ranges approximately from 60 to $100 \mathrm{~ms}$ (Chan et al., 1979). However, if a delay of longer than $4 \mathrm{~ms}$ was applied to the delayed response model, the model could not walk naturally, as was the case in a previous study (Hase and Obinata, 2002). One reason was that the proposed model did not have a mechanism to compensate for the delay. Some computational biological studies have mentioned the mechanism stabilizing walking pattern with respect to the neuronal delay properties (Ohgane et al., 2004). The specific power for locomotion increased greatly in the delayed response model, although the basic walking factors such as walking speed did not change significantly because the muscular activation pattern vibrated with high frequency as shown in Figure 3 due of the influence of the delay property.

In control engineering technology, the feedback control is influenced by the delay properties, but the feedforward control is not. Walking motion is not affected by the neuronal delay if it is controlled based on the feedforward control mechanism in which pre-programmed motor commands control muscle activities without sensory feedback signals from the somatic senses. Therefore, walking motion in actual humans may be controlled by a combination of the feedback control based on the somatic senses and the feedforward control based on pre-programmed motor commands in the brain, so that both stability with respect to the delay and adaptability to perturbations can be handled, although this control model is only hypothetical. In addition, it is hypothesized that the function of such a compensation mechanism for the delay may be reduced with age, similar to the fact that the muscular latency time in older adults during walking was significantly longer than that of younger adults, as mentioned earlier (Hase et al., 2002). In any case, the delayed response or lowering of functions compensating for the delay with age is thought to be a strong cause of unstable, inefficient walking.
Second, weak muscle function has a bad influence on walking pattern. In particular, the step length is strongly related to muscle weakness. Here, let us compare bipedal walking with a simple forced vibration, because bipedal locomotion is assumed to be similar to pendulum vibration. Vibration engineering shows that the amplitude of a forced vibration is proportional to the magnitude of the driving force. Considering the step length of walking as the amplitude of vibration and the muscle force as a driving force for vibration, reduction of the muscle force is thought to cause the reduction in the step length. On the other hand, in general, walking motion does not always require strong muscle power because the basic walking pattern is effectively realized through gravity by a pendulum motion (McGeer, 1990). Peaks of muscle force during walking do not always reach the maximum capacity of the muscle even in older adults. Thus, muscle weakness is not always a principal factor in unstable walking, although it does shorten step length. As such, muscle weakness should be a principal factor for extremely weak older adults who are unable to stand up by themselves.

Third, the inclined posture and joint contracture also had a bad influence on the walking pattern. However, these did not appear to be principal factors. The inclined posture lengthened the walking cycle and increased the step length. Since such phenomena are contrary to the short-stepped gait that is typical in older adults, the inclined posture was estimated not to be a major factor. In the joint contracture model, the joint elastic coefficient was set to be five times greater than that of the intact model in order to clarify the influence of the joint contracture. However, passive joint resistance in actual humans does not always change so significantly, and is usually within approximately $30 \%$ (Research Institute of Human Engineering for Quality Life, 2000). Moreover, the influence of joint contracture on walking pattern is not always significant compared to other malfunctions in the hypothesized models. Therefore, these influences on walking 
are thought to have been secondary.

In summary, the physical causes of instability and falling risk in older adults are hypothesized to be as follows: primary, the delay response or poor compensation mechanism of the delay; secondary, weak muscle function; subsidiary, changes in posture and joint contracture. This sequence should be considered when planning walking rehabilitation.

\section{Advantages of the computer simulation method}

The important factor of the present simulation method was that only one body parameter, such as maximum muscle force, was varied in each model, so that the influence of each parameter on walking could be analyzed independently. Although in the actual case several body functions are reduced simultaneously with age, computer simulation methods are able to simplify such complex relations between body functions and motions.

Computer simulation methods also have various possible applications for biomechanical and anthropological studies. One application is the synthesis of difficult or impossible conditions that cannot be realized in actual experiments, particularly for older adults. For example, computer synthesis of falling motion will be useful for clinical assessment in order to prevent falling, or fractures related to falling. Computer simulation techniques may also be helpful in longitudinal studies such as the growth and evolution of humans, because computer simulation can quickly reproduce or predict such longitudinal processes if the dynamics of these processes are modeled mathematically. It is also important to simulate the process of aging for locomotion or other types of human motion in a computer. The same concept will be applied to clinical assessment. That is, in order to predict the process of rehabilitation of walking, effective treatment can be prescribed without burdening the patient.

\section{Future studies}

Validation of the simulation results is paradoxical in a sense. The walking pattern generated by the hypothetical models seems to represent the characteristics of the walking pattern in older adults, e.g. shorter step length in the weak muscle model. However, it is difficult to compare directly the simulation results obtained by hypothetical models with the experimental data colleted from actual older adults because the hypothetical model has the property that only the body parameter was varied, although in reality several body functions are reduced simultaneously with age. Such computer simulation methods have both advantages and disadvantages. One advantage of this simulation is the ability to investigate difficult or impossible conditions that cannot be realized in actual experiments, particularly for older adults. In contrast, one disadvantage of this method is that the simulation results are not validated by comparison with the experimental data. Comparing the other walking simulation models, we believe our simulation model has some advantages. However, there is still a gap between the computer model and actual humans. The fundamental ability of the walking simulation model should be improved to realize a valid walking pattern. The problem should be solved from the both sides of the mechanical control theory, i.e. from the points of view of both robotics and neurophysiology. There- fore, the specific characteristics and limitations of the simulation results should be considered when the simulation method is used.

Unstable walking and motion in older adults is caused not only by the musculoskeletal factor investigated herein, but also by psychological problems such as the reduction of cognition and attention. The importance of these problems and the difficulty in dealing with them through computational approaches such as that presented herein are acknowledged. However, these problems will hopefully be examined in the future.

\section{Acknowledgments}

The present study was supported by a Grant-in-Aid for Scientific Research from the Japan Society for the Promotion of Science (JSPS).

\section{References}

Chan C., Melvill J.G., Kearney R., and Watt D. (1979) The 'late' electromyographic response to limb displacement in man. I. Evidence for supraspinal contribution. Electroencephalography and Clinical Neurophysiology, 46: 173-181.

Furuna T., Nagasaki H., Nishizawa S., Sugiura M., Okuzumi H., Ito H., Kinugasa T., Hashizume K., and Maruyama H. (1998) Longitudinal change in the physical performance of older adults in the community. Journal of the Japanese Physical Therapy Association, 1: 1-5.

Hase K. and Obinata G. (2002) Computer simulation study of human locomotion with a three-dimensional entire-body neuro-musculo-skeletal model. II. Biomechanical relationship between walking stability and neuro-musculo-skeletal system. JSME International Journal, Series C, 45: 1051-1057.

Hase K. and Obuchi S. (2002) Computer simulation study of human locomotion with a three-dimensional entire-body neuro-musculo-skeletal model. III. Simulation of pathological walking and its application to rehabilitation engineering. JSME International Journal, Series C, 45: 1058-1064.

Hase K. and Yamazaki N. (2002) Computer simulation study of human locomotion with a three-dimensional entire-body neuro-musculo-skeletal model. I. Acquisition of normal walking. JSME International Journal, Series C, 45: 1040-1050.

Hase K. and Yokoi T. (2002) Computer simulation study of human locomotion with a three-dimensional entire-body neuro-musculo-skeletal model. IV. Simulation of running motion and its transition process. JSME International Journal, Series C, 45: $1065-1072$.

Hase K., Obuchi S., and Horie T. (2002) Rehabilitation system to prevent falls during walking in older adults (Planning of design concept and primary experiments). Transaction of the Japan Society of Mechanical Engineers, 68: 1245-1250 (in Japanese).

Hase K., Miyashita K., Ok S., and Arakawa Y. (2003) Human gait simulation with a neuro-musculo-skeletal model and evolutionary computation. Journal of Visualization and Computer Animation, 14: 73-92.

Kaneko M., Fuchimoto K., Fuchimoto T., Morimoto Y., Kimura M., Kitamura T., Tsutsui Y., and Arai T. (1990) Biomechanical analysis of walking and fitness testing in elderly women. In: Kaneko M. (ed.), Fitness for the Aged, Disabled, and Industrial Worker. Human Kinetics Books, Champaign, IL, pp. 84-89.

McGeer T. (1990) Passive dynamic walking. International Journal of Robotics Research, 9: 62-82.

Murray M.P., Kory R.C., and Clarkson B.H. (1969) Walking pat- 
terns in healthy old men. Journal of Gerontology, 24: 169178.

Nishizawa S., Furuna T., Sugiura M., Okuzumi H., Nagasaki H., Itoh H., Fujita M., Ogiue M., and Ueda Y. (2000) Walking parameters that strongly characterize that gait of older adults. In: The Society of Biomechanisms Japan (ed.), Biomechanisms 15. University of Tokyo Press, Tokyo, pp. 131-140 (in Japanese).

Ohgane K., Ei S., Kudo K., and Ohtsuki T. (2004) Emergence of adaptability to time delay in bipedal locomotion. Biological Cybernetics, 90: 125-132.

Research Institute of Human Engineering for Quality Life (2000) Report of "Measurement and Evaluation of the Human Dynamic Characteristics", Research Institute of Human Engineering for Quality Life, Osaka (in Japanese).

Ralston H.J. (1976) Energetics of human walking. In: Herman R.N., Grillner S., Stein P.S., and Stuart D.G. (eds.), Neural Control of Locomotion. Plenum Press, New York, pp. 77-98.

Taga G., Yamaguchi Y., and Shimizu H. (1991) Self-organized control of bipedal locomotion by neural oscillators in unpredictable environment. Biological Cybernetics, 65: 147-159.

Taga G. (1995) A model of the neuro-musculo-skeletal system for human locomotion. Biological Cybernetics, 73: 113-121.

\section{Appendix}

Figure A1 shows the kinematic and dynamic data obtained from the intact simulation model: the muscle forces, the joint moments, the joint angles, and the ground reaction forces during one walking cycle, which have been published in a previous paper (Hase and Yamazaki, 2002). The data presented here are from walking at a speed of $1.39 \mathrm{~m} / \mathrm{s}$, a stride length of $1.52 \mathrm{~m}$, a step width of $0.20 \mathrm{~m}$, and a walking cycle time of $1.09 \mathrm{~s}$.

In general, the simulated walking pattern agrees closely with that of an actual human. For example, the vertical ground reaction force has a two-peak waveform, which is one of the characteristics of normal walking. Basic cadence parameters, such as walking speed, correspond well to those of the average human gait. However, some differences between the simulation results and actual human walking motion are also observed. For example, the extensor moment on the hip joint is relatively higher and the extensor moment on the knee joint during the stance phase is lower compared to those of an actual human. Moreover, the intact simulation model was intended for to be applied to the synthesis of the normal walking pattern of younger adults and not that of older adults. 


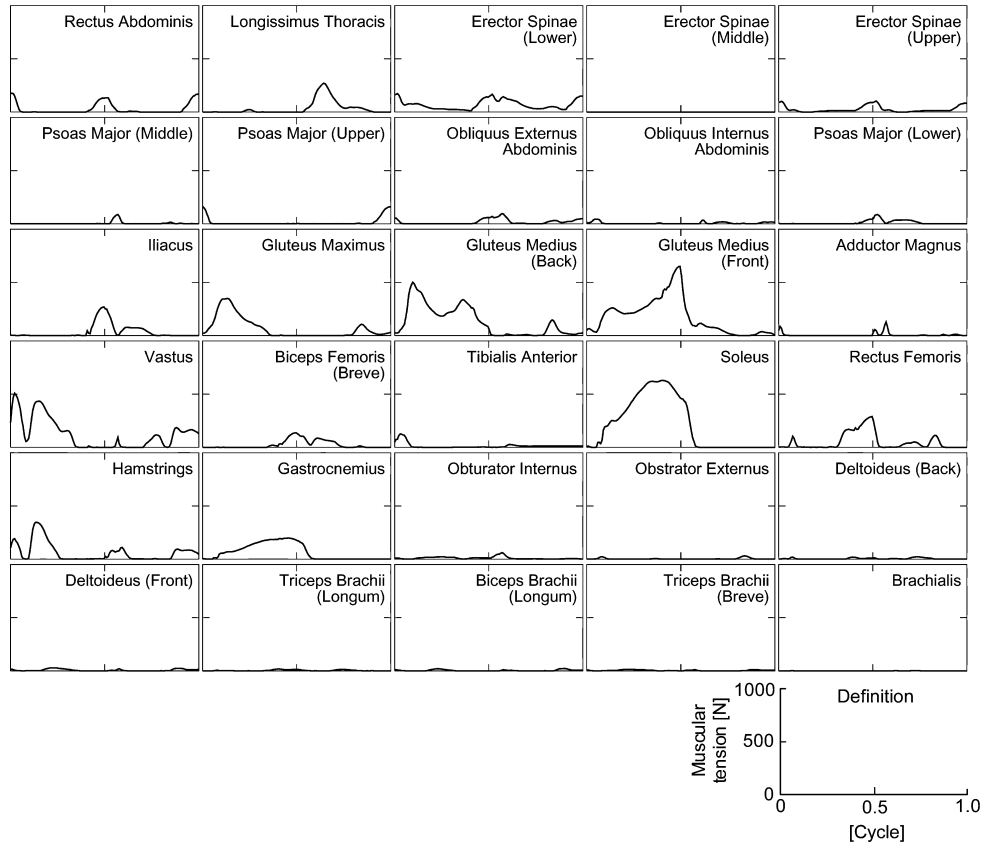

(a) Muscle forces

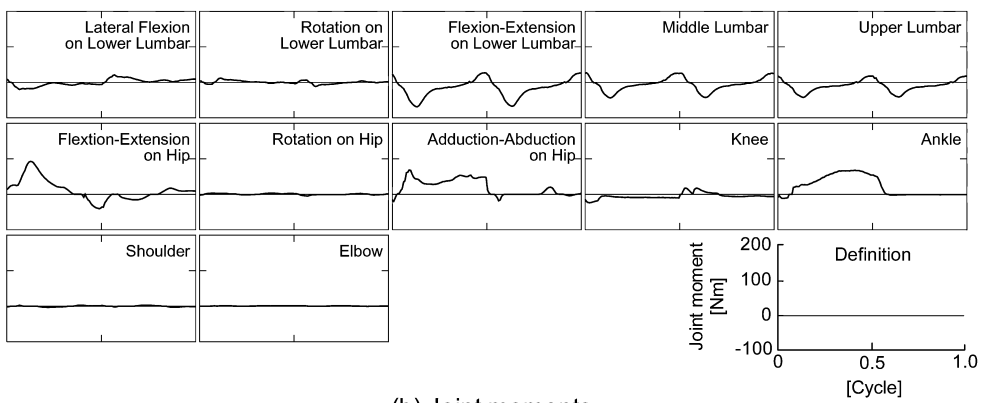

(b) Joint moments

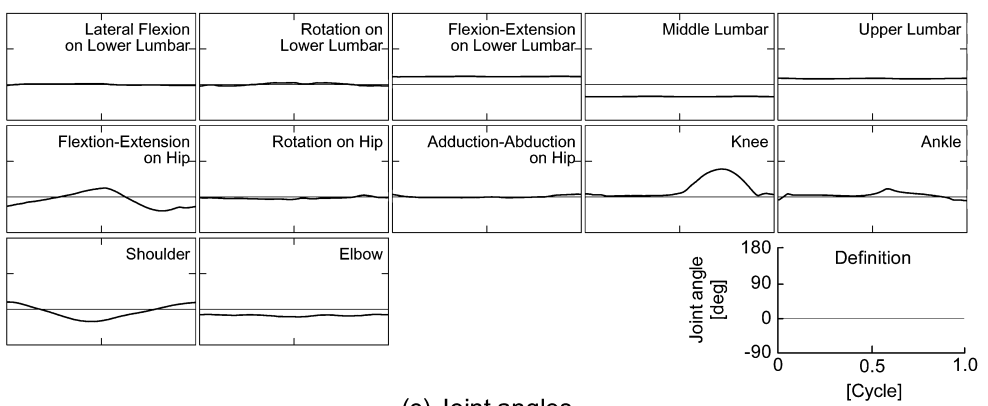

(c) Joint angles
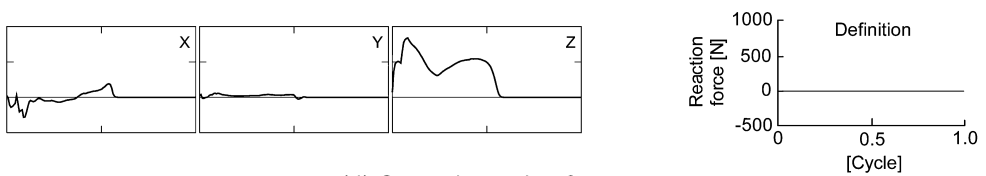

(d) Ground reaction forces

Figure A1. Kinematic and dynamic data obtained from the intact simulation model. (a) Muscle forces, (b) joint moments, (c) joint angles, (d) ground reaction forces. For all of the graphs, time is normalized by the time required for one walking cycle, where 0 and 1 indicate the points at which the right heel touches the ground. For the muscle forces and ground reaction forces, the data related to the right side of the body are illustrated. For the joint moments and angles, the data with respect to the left upper and lower extremities are omitted. The positive values of the joint moment and angle show clockwise movements. 